\title{
INSTRUMENTATION ET COMPORTEMENT DE PIEUX D'ACCOSTAGE SOUS FAIBLE CHARGE HORIZONTALE
}

\author{
Daniel LEVACHER, Salim MEZAZIGH \\ GRGC, Laboratoire de Mécanique des Fluides et Génie Civil, Université du Havre
}

E. VASSELIN

Labo. d'Analyse et de Commande des Systèmes LACOS - Université du Havre

\author{
J.M. SEVIN \\ ETI, Port Autonome de Nantes - St Nazaire
}

\begin{abstract}
Résumé
Le présent article décrit l'instrumentation de trois pieux d'accostage faisant partie du terminal de Cheviré (Port Autonome de Nantes - Saint-Nazaire). Une fois l'instrumentation réalisée à terre, les toix pieux tubulaires ouverts de $28 \mathrm{~m}$ de long et de $1,22 \mathrm{~m}$ de diamètre ont été battus pour une fiche moyenne de $15 \mathrm{~m}$. Un bilan de l'instrumentation a été dressé après le battage de ces pieux. Une connectique appropriée a été mise en place de façon à faciliter l'acquisition des mesures extensométriques. Les trois pieux ont fait l'objet de chargements statiques horizontaux de faible amplitude (équivalente au 1/10è de la charge de service). De ces essais de chargement, nous avons pu tirer les éléments nécessaires à la justification dizune telle instrumentation. Les mesures extensométriques effectuées durant les essais ont notamment permis d'obtenir la distribution des moments fléchissants. Des mesures de déplacement en tête de pieu ont été également entreprises à l'aide de divers moyens de mesures (théodolite, télémètre et caméra). Dans l'ensemble, ces essais ont donné satisfaction et permis d'envisager un complément d'études du comportement de ces pieux soumis à une forte charge horizontalè.
\end{abstract}

Mots-clés : pieu, charge horizontale, essai en vraie grandeur, déplacement, moment fléchissant

\section{Introduction}

La réalisation d'aménagements portuaires (terminaux, quais, ducs d'Albe...) nécessite très souvent l'implantation de pieux battus sollicités horizontalement. Si le concepteur dispose de plusieurs méthodes de dimensionnement, il n'en demeure pas moins que les difficultés résident le plus souvent dans le choix des hypothèses de calcul : pieu flexible ou rigide, ancrage en pied ou non, longueur de transfert, calcul des modules de réaction, courbes $\mathrm{p}-\mathrm{y}$, utilisation de coefficients réducteurs (effet de proximité de talus, de surcharges dues à la présence de remblais, d'interaction dans le cas de rangées de pieux). Le mode de mise en cuvre des pieux influence très peu le comportement en chargement latéral. 
Devant le nombre important de facteurs à retenir et en fonction de certaines incertitudes sur les choix, le concepteur a recours à l'utilisation de coefficients de sécurité élevés et ceci a tendance à surdimensionner les pieux. Cependant, le comportement de pieux battus chargés latéralement reste difficile à appréhender et doit susciter de nouvelles études en vue d'améliorer la compréhension du phénomène et de développer des formules ou des logiciels de calcul adaptés. Ces études doivent concourir à une nécessaire confrontation entre analyses théoriques et expérimentation (simulation physique et/ou essai en vraie grandeur).

\section{Présentation de l'étude}

L'enjeu

Le Port Autonome de Nantes - St Nazaire se doit de développer ses installations portuaires afin d'accueillir un nombre croissant de bateaux et navires transportant divers matériaux et marchandises, en différents endroits de la Loire. Chaque installation constitue un terminal [fig. 1], ainsi dans la partie portuaire de Nantes-Cheviré, on retrouve deux terminaux voisins : le terminal à bois et le terminal sablier [fig. 2]. Selon la nature des matériaux et la taille des navires, la conception du terminal peut être réduite à une rangée de pieux d'accostage derrière lesquels est contruit un appontement d'accès. C'est le cas du terminal sablier composé d'une rangée de trois pieux d'accostage.

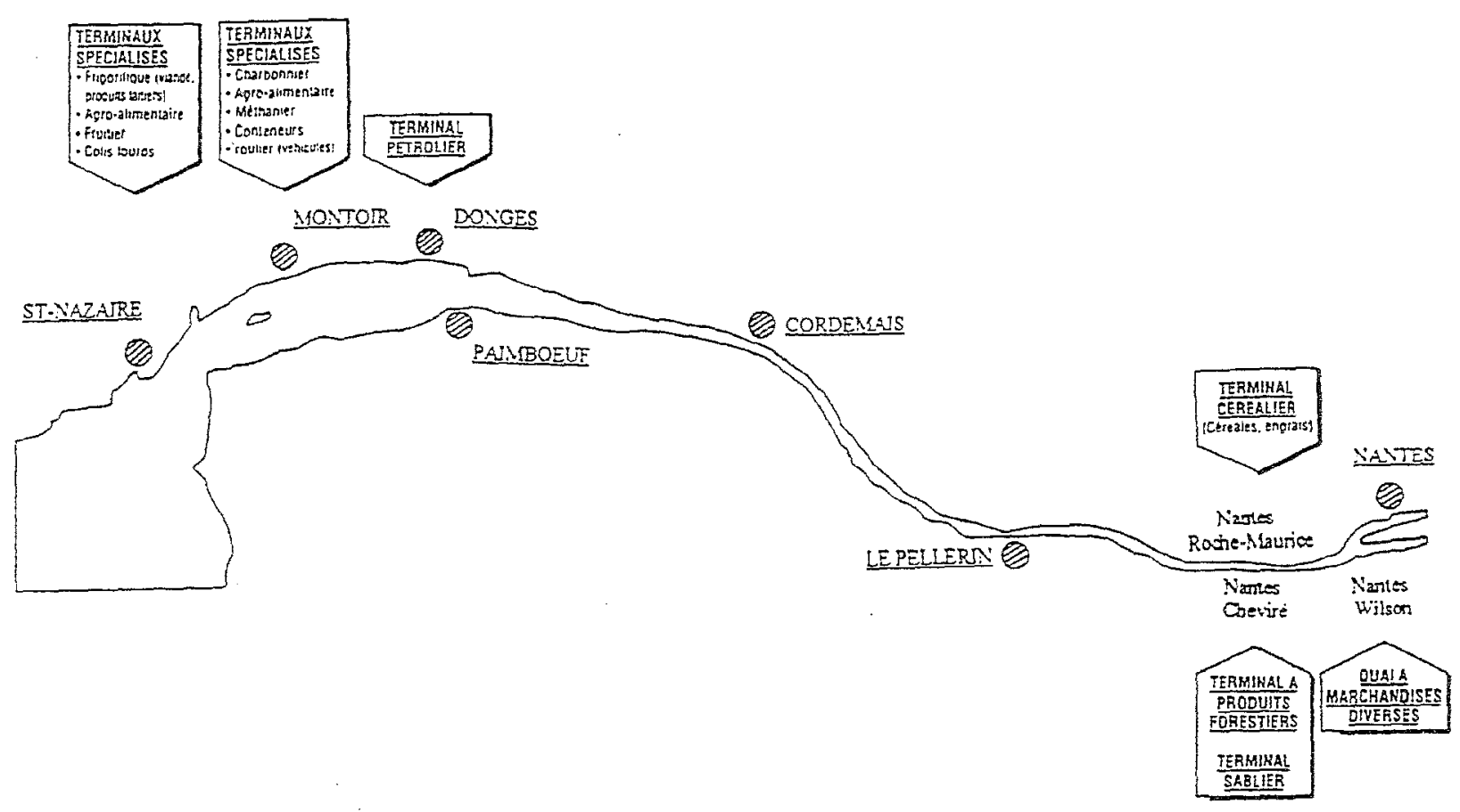

Fig. 1 : Les terminaux du Port Autonome de Nantes - St Nazaire 


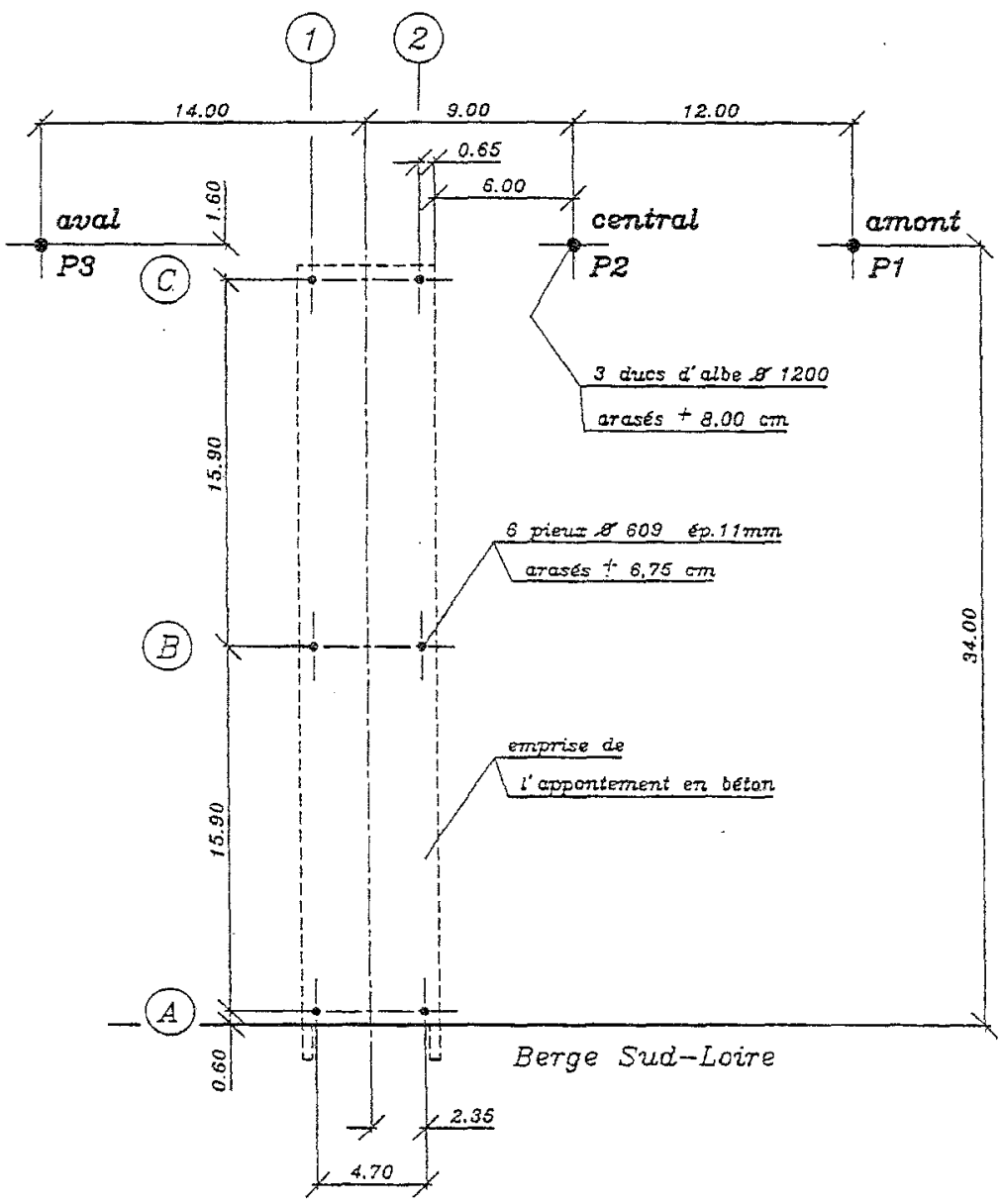

Fig. 2 : Le terminal sablier de Cheviré

\section{L'obiectif}

Les essais de pieux décrits dans cet article ont été réalisés sur le site opérationnel de Cheviré, pour de faibles chargements latéraux. L'instrumentation requise pour l'expérimentation a été faite à terre avant l'installation par battage des pieux d'accostage. Cette phase préliminaire, a constitué, à elle seule, une importante partie de l'étude dans la mesure où, en un court délai, il a fallu équiper l'ensemble des trois pieux, de dix sections de mesures soit un total de 120 jauges de déformations[fig. 3]. Le type de pieu retenu - tubulaire ouvert - et le mode de mise en place - le battage - ont imposé un type de protection renforcé des jauges afn de garantir au mieux l'instrumentation après battage. Par la suite, les essais de chargement ont permis d'une part de contrôler l'état de cette instrumentation et d'aume part d'obtenir des distributions des moments fléchissants dans les pieux soumis à une charge latérale d'environ $50 \mathrm{kN}$. L'ensemble des résultats des essais font l'objet d'un rapport final [réf. 1]. Ces essais ont aussi comme but une meilleure connaissance du comportement des pieux sous charges horizontales et l'évaluation des méthodes de calcul. 


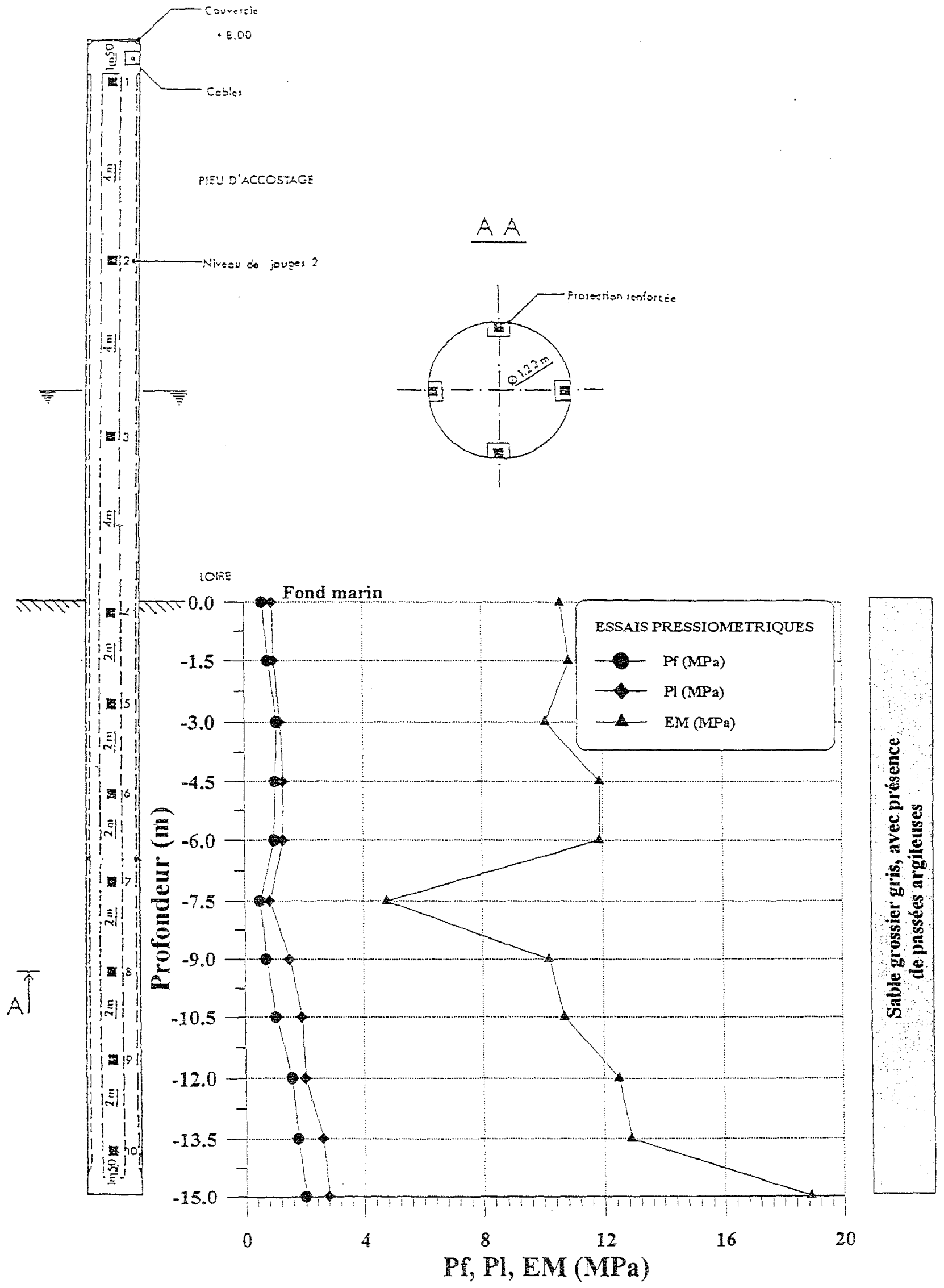

a) Pieu instrumenté

b) Profils pressiométriques et descriptif du fond marin

Fig. 3 : Réparition des sections de mesures pour un pieu et données géotechniques 


\section{L'équipement des pieux en instrumentation}

Les jauges

Elles sont de type CEA-06-250-UN-120 foumies par VISHAY MICROMEASURMENTS DIVISION. Elles sont encapsulées et à sorties renforcées. Elles ont été collées avec des cosses-relais pour la connexion. L'installation s'est effectuée sur le pieu posé à l'horizontal sur un quai.

\section{Les colles et protections légères}

Successivement après avoir conditionné la surface métallique intérieure du pieu (ponçage, dégraissage et neutralisation), les jauges ont été collées (colle AE 10) puis isolées électriquement contre l'humidité avec du M-Coat-A et protégées mécaniquement avec une résine de type PR1422B2.

Lecâblage

Des câbles blindés à trois fils ont été utilisés. L'un des fils est soudé sur une borne de la cosse-relai, les deux autres sur la seconde borne de manière à ce que la résistance des fils puisse être prise en compte par le conditionneur. Les câbles sont maintenus contre la paroi intérieure des pieux par la résine (PR) en altemance tous les mètres avec des plots adhésifs. Les câbles sont organisés en torons vers la tête du pieu et les deux demiers mètres ont été entièrement protégés avec de la gaine annelée blindée (CAPRIPLAST).

\section{Les protections métalliques}

Afin de protéger les jauges lors du transport et surtout lors de la mise en position du pieu (vibro-battage et battage), des cornières d'acier ont été placées sur chaque génératrice à l'aplomb des torons. Il s'agit de cornière $60 \times 60 \times 6 \mathrm{~mm}$. Un biseau sur les comières a été réalisé au niveau de la partie inférieure du pieu pour favoriser la pénétration dans le sol. Elles sont à ce niveau soudées sur plus d'un mètre puis les cordons de soudure d'environ $10 \mathrm{~cm}$ s'alternent de chaque coté de la comière jusqu'à la tête du pieu. Des étriers de renfort au nombre de 20 sont placés au-dessus de la cornière. Les dimensions de ces étriers sont les suivantes : $75 \times 75$, longueur $150 \mathrm{~mm}$, épaisseur $8 \mathrm{~mm}$.

\section{La mise en attente des câbles}

Les torons ont été lovés dans des sceaux de stockage en acier soudés en tête de pieux. Ces seaux sont de diamètre $0,339 \mathrm{~m}$, de longueur $0,8 \mathrm{~m}$ et ils ont une épaisseur de $12 \mathrm{~mm}$.

\section{Les vérifications}

Toutes les lignes de mesure (câble plus jauge) ont été testées (mesure de résistance et de continuité électrique) avant l'installation des comières de protection par soudure. Aucune anomalie n'a alors été décelée.

Des vérifications identiques après soudure ont montré qu'une seule jauge était défaillante. Soit 1 sur 120 , ce qui paraît tout à fait correct et ne remet pas en cause et la technique de protection et les mesures attendues.

\section{Conclusions}

Le travail d'instrumentation des trois pieux d'accostage a consisté en la mise en cuvre sur site de techniques de mesures essentiellement utilisées en laboratoire. Il en résulte que 120 jauges de déformation ont été mises en place reliées par près de $4 \mathrm{~km}$ de câbles. 


\begin{tabular}{|c|c|c|c|}
\cline { 2 - 4 } \multicolumn{1}{c|}{} & $\begin{array}{c}\text { PIEU AVAL } \\
\text { Pieu n }^{\circ} 3\end{array}$ & $\begin{array}{c}\text { PIEU CENTRAL } \\
\text { Pieu n }{ }^{\circ}\end{array}$ & $\begin{array}{c}\text { PIEU AMONT } \\
\text { Pieu n }{ }^{\circ}\end{array}$ \\
\hline \begin{tabular}{c} 
ongueur totale de câblè $(\mathrm{m})$ \\
\hline $\begin{array}{c}\text { Longueur en attente } \\
(\mathrm{m})\end{array}$
\end{tabular} & 1252 & 1132 & 1612 \\
\hline
\end{tabular}

\section{L'acquisition des mesures extensométriques}

Le dispositif (figure 4)

Les torons de chaque génératrice se regroupent en un toron principal (de 40 câbles) ce qui facilite les manipulations et limite les omissions de connexion. Des boites relais sont fixées à la passerelle d'accès aux pieux (coffrets étanches SAREL THALASSA IP56), elles protègent les prises des torons contre l'eau et les agressions marines et permettent un montage-démontage rapide de l'installation. Les prises sont de type DL (à insertion nulle) ITT CANON de 156 contacts. Elles offrent un contact immédiat avec les conditionneurs VISHAY (svstèmes 2100).

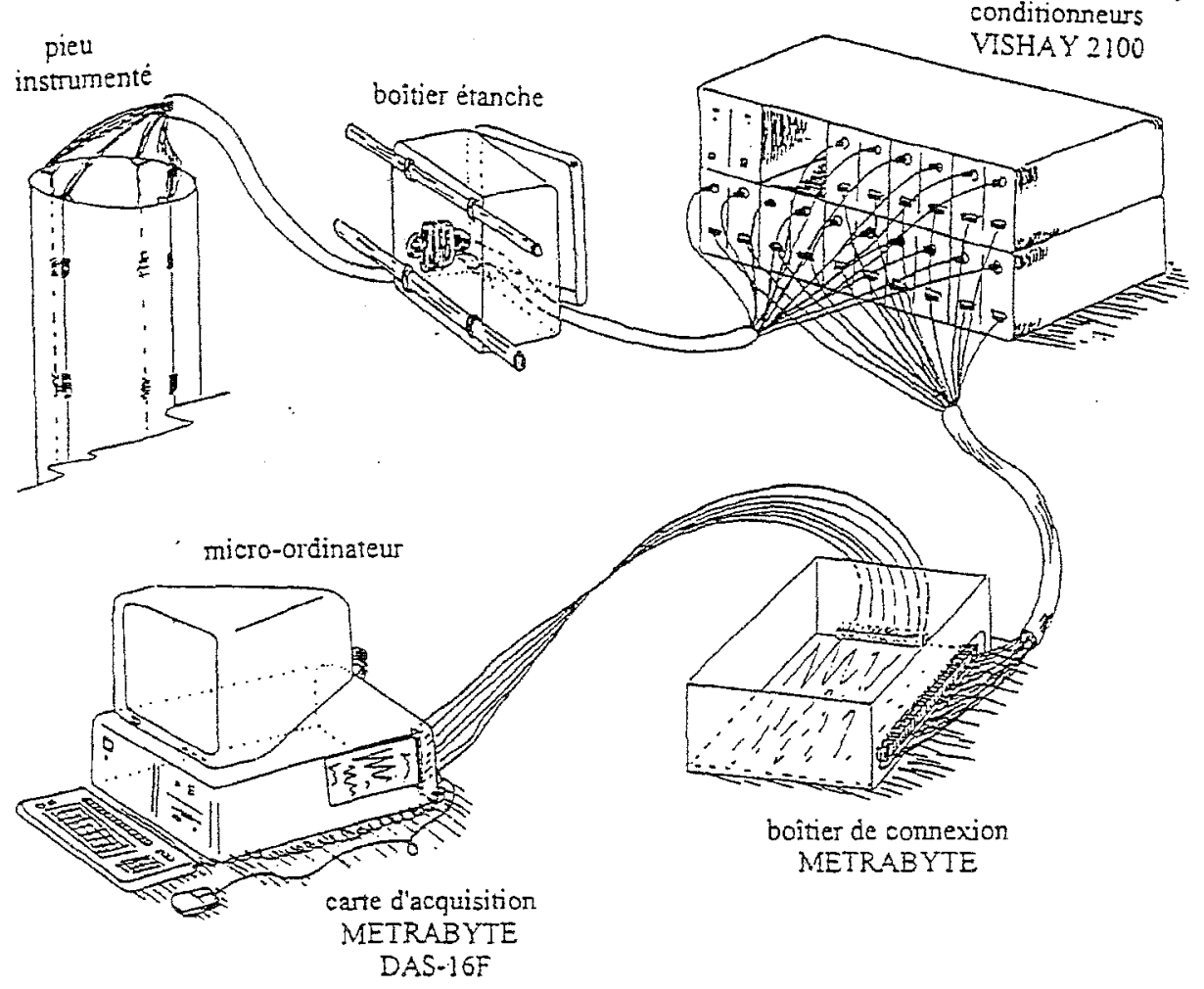

Fig. 4 : Dispositif d'acquisition de mesures sur site

Une carte d'acquisition analogique multivoies et multifonctions (METRABYTE-KEITHLEY DAS-16F) assure l'interface avec un microordinateur. Le convertisseur analogique-numérique de cette carte travaille à la fréquence maximale de $100 \mathrm{kHz}$. Le micro-ordinateur de type $\mathrm{PC}$, va permettre d'optimiser I'acquisition, le stockage et le traitement des données. 
L'acouisition des mesures

Les déformations décelées par les jauges génèrent au niveau du montage $1 / 4$ de pont de faibles variations de tension (quelques $\mathrm{mV}$ ). Ce signal est filué et amplifié par les conditionneurs et l'information est transmise à la carte sous forme d'une tension variable bipolaire de haut niveau (juqu'à $\pm 10 \mathrm{~V}$ ).

Ces signaux sont traités et enregistrés par le programme "PIEUX" conçu spécialement sous ASYST, environnement de développement dédié à l'acquisition et au traitement des données. II permet d'acquérir les signaux, de les stocker, d'effectuer des traitements mais également de les visualiser immédiatement.

\section{Résultats des essais de chargement}

Déplacements

Des mesures de déplacement en tête ont été réalisées à l'aide de théodolite classique, de télémètre et de caméra. L'objectif de ces mesures n'était pas de construire les courbes charge-déplacement mais de mettre différents dispositifs de mesure en place en prenant en compte les conditions environnementales de l'essai et de tester leur performance. Le tableau I donne un ordre de grandeur du déplacement mesuré pour une charge comprise entre 38 et $41 \mathrm{kN}$.

\begin{tabular}{|c|c|c|l|}
\hline Pieu & $\begin{array}{c}\text { Effort appliqué } \\
(\mathrm{kN})\end{array}$ & $\begin{array}{c}\text { Déplacement en } \\
\text { tête mesuré }(\mathrm{mm})\end{array}$ & Moyen de mesure \\
\hline \multirow{3}{*}{ Central } & 40.9 & 19.6 & Théodolite classique \\
& 41.1 & 21.1 & Théodolite classique \\
& 40.2 & 17.0 & Télémètre \\
& 38.0 & 18.8 & Caméra \\
\hline \multirow{3}{*}{ Amont } & 40.9 & 25.4 & Théodolite classique \\
& 41.1 & 18.0 & Théodolite classique \\
\hline \multirow{3}{*}{ Aval } & 40.2 & 19.0 & Télémètre \\
& 39.4 & 18.9 & Caméra \\
& 39.8 & 21.0 & Caméra \\
& 40.8 & 17.8 & Caméra \\
\hline
\end{tabular}

Tab. I: Valeurs mesurées du déplacement en tête

Les trois moyens de mesure utilisés donnent des valeurs qui varient de 17 à $25.4 \mathrm{~mm}$ dont la valeur moyenne est égale à $19.8 \mathrm{~mm}$. Ces appareillages conviennent pour le type de chargement effectué par palier. Dès lors qu'il s'agit d'essai de chargement en continu, seule la caméra pourra effectuer le suivi du mouvement du pieu par saisie d'images. La limitation éventuelle sera la fréquence d'acquisition des images en fonction de la vitesse de chargement.

\section{Moments fléchissants}

Les moments fléchissants sont obtenus directement à partir de la mesure des déformations sur site. Ainsi en fonction de la charge appliquée en tête, on représente la variation des moments fléchissants en fonction de la profondeur. Ces courbes de variation des moments fléchissants foumies par les mesures montrent une similitude du comportement des pieux en profondeur. On remarque notamment que le moment maximal se situe entre -0.50 et $-2.00 \mathrm{~m}$. Il atteint une valeur 
comprise entre 0.70 et $0.80 \mathrm{MN}$.m pour un effort en tête de $40 \pm 2 \mathrm{kN}$. La figure 5 donne la distribution des moments pour le pieu amont, à titre d'exemple.

Valeur des charges appliquées en tête

$\begin{array}{rll} & 015.0 \mathrm{kN} & \Delta \\ -8.0 \mathrm{kN} & -21.9 \mathrm{kN} & -35.5 \mathrm{kN}\end{array} \quad \therefore 41.8 \mathrm{kN}$

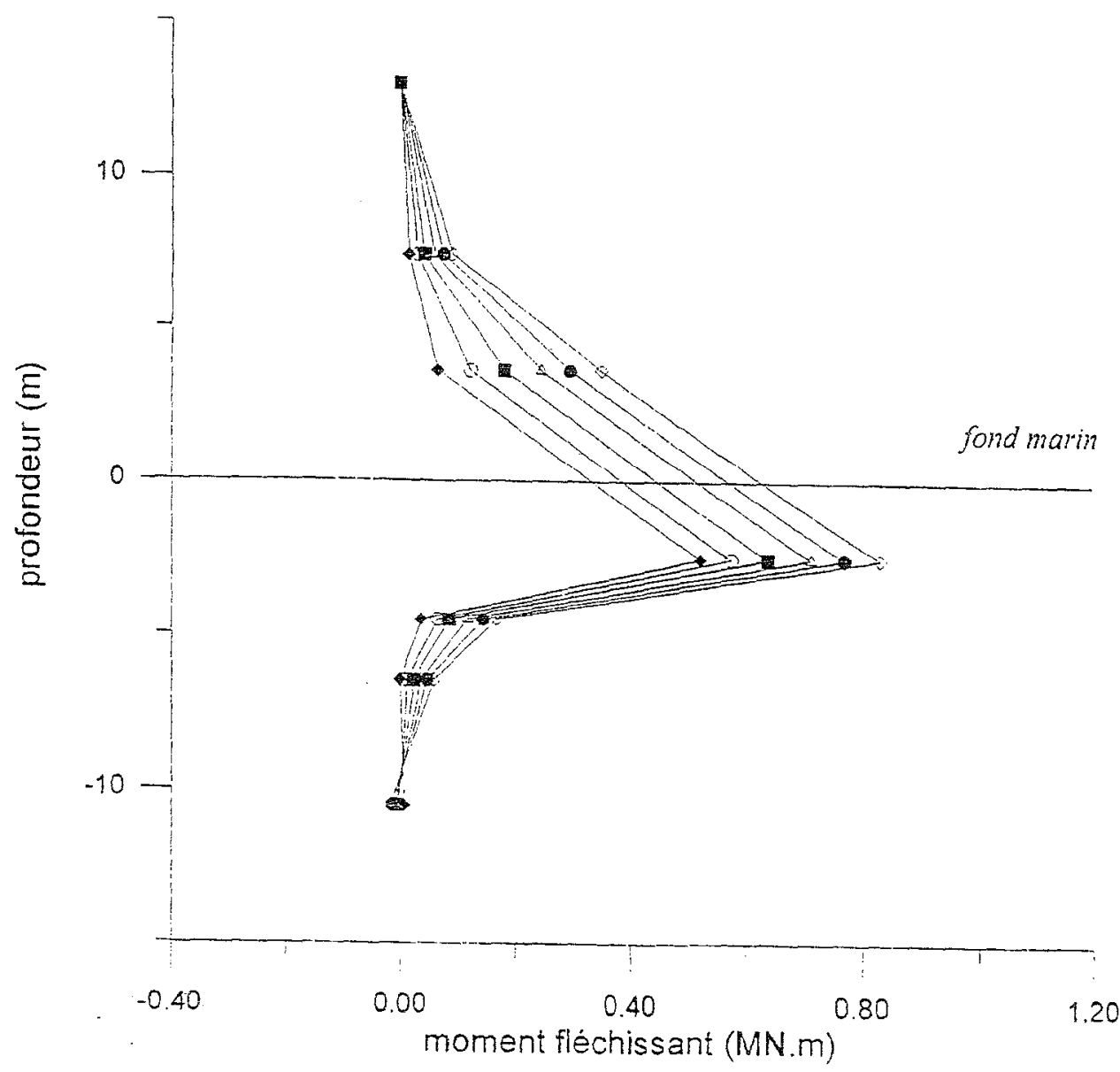

Fig. 5 : Moments résultants mesurés pour le pieu amont - Génératrice $n^{\circ} 2-6$ sections de mesures

Le pieu aval ( $n^{\circ} 3$ ), le mieux instrumenté, a donné des résultats corrects comme l'attestent les courbes (voir figure 6) des moments fléchissants obtenus sur deux génératrices opposées (référencées 2 et 4 , réf. 1). Ces valeurs résultent uniquement du chargement latéral appliqué. Les effets dus à l'effort normal et à la température sont éliminés. 


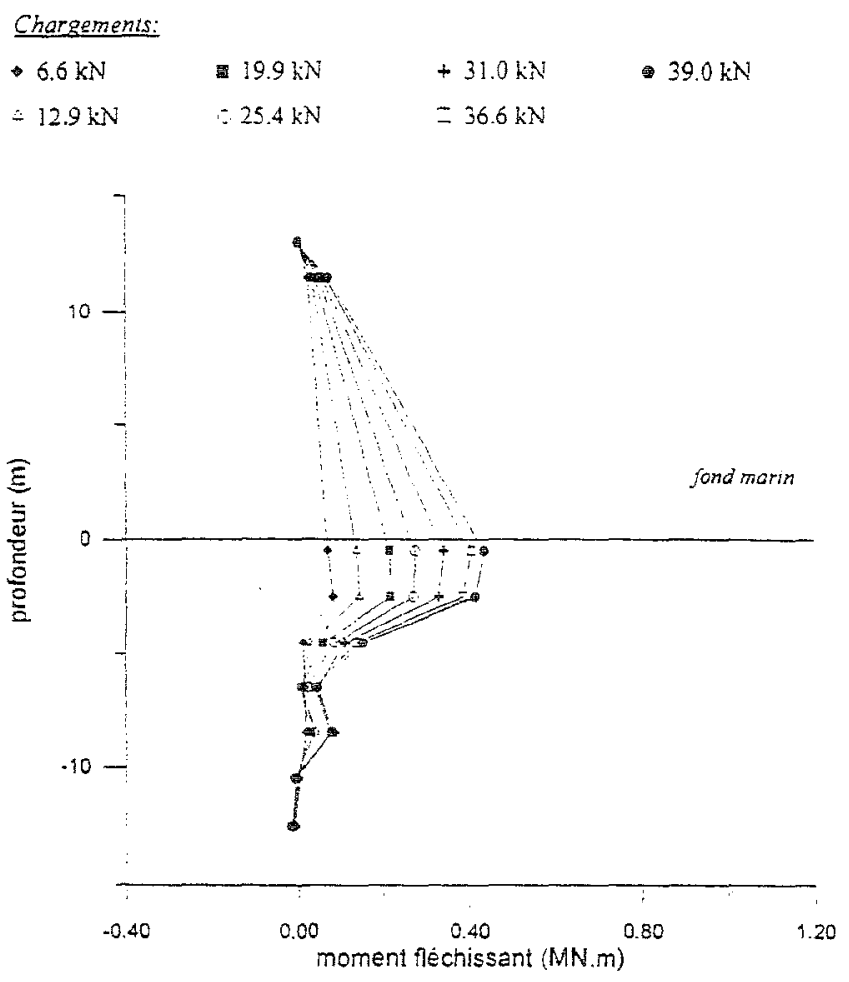

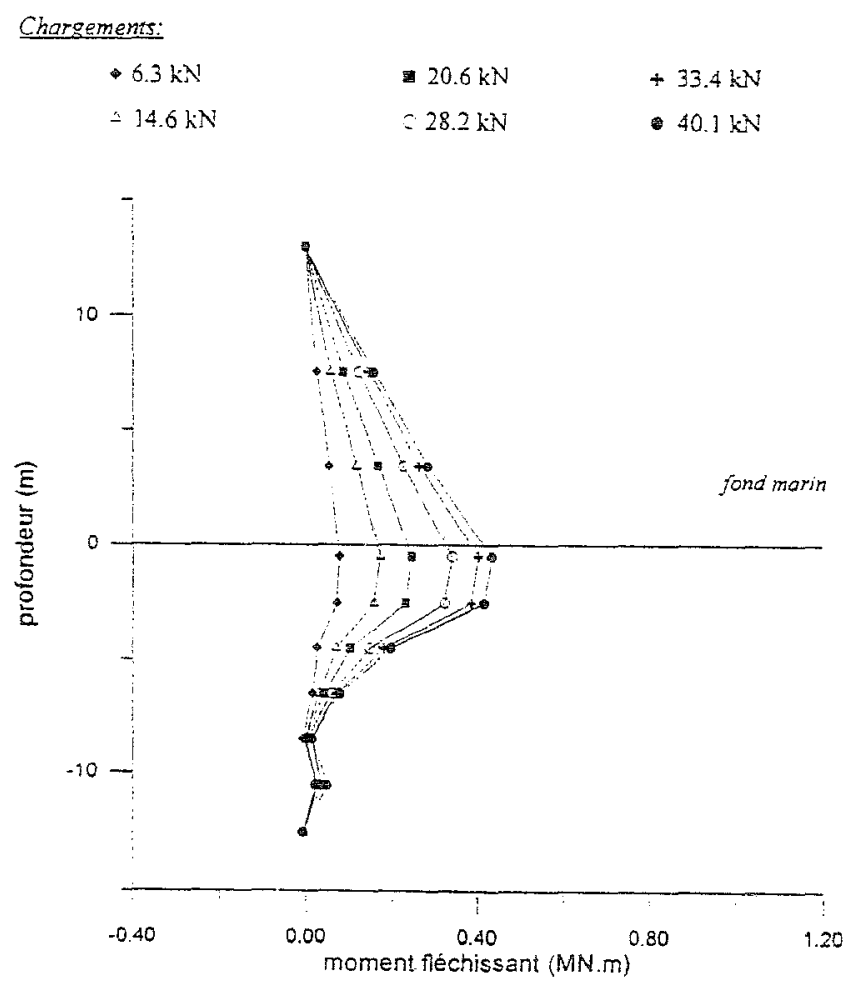

a) Génératrice 2

b) Génératrice 4

Fig. 6 : Moments fléchissants mesurés sur deux génératrices opposées du pieu aval

Confrontation avec une méthode de prévision

En ce qui concerne la méthode de prévision, nous avons utilisé ici le logiciel PIIATE du LCPC [Réf. 2] basé sur le calcul aux modules de réaction. Il permet le calcul d'un pieu isolé, fiché dans un sol découpé en tranches horizontales, chacune étant caractérisée par une courbe de réaction p-y non linéaire quelconque. La courbe de réaction pour le pieu, à un niveau donné est établie à partir des résultats pressiométriques à ce niveau.

La simulation numérique du comportement des pieux d'accostage a été entreprise en vue de calculer les déplacements et les moments fléchissants le long du pieu soumis à un chargement latéral donné. Les courbes de réaction ont été déduites des caractéristiques pressiométriques du site (fig. 3b). Elles sont de type bi-linéaire, et établies à partir des valeurs des pressions limites P1. Les conditions aux limites imposées en pied sont celles d'un pieu libre. Une comparaison a été faite entre les déplacements calculés et mesurés en tête dont les valeurs sont récapitulées dans le tableau II. 
SESSION IV : Innovations technologiques en Génie Côtier

\begin{tabular}{|c|c|c|}
\hline $\begin{array}{c}\text { Effort latéral appliqué } \\
(\mathrm{kN})\end{array}$ & $\begin{array}{c}\text { Péplacement en tête mesurespéplacement en tête calculè } \\
(\mathrm{mm})\end{array}$ & $(\mathrm{mm})$ \\
\hline 39.8 & $20.85^{*}$ & 23.5 \\
40.2 & $18^{*}$ & 23.7 \\
40.8 & 17.8 & 24.1 \\
40.9 & $22.5^{*}$ & 24.1 \\
41.1 & 19.55 & 24.2 \\
\hline
\end{tabular}

Tab. II : Valeurs comparées des déplacements en tête

(*valeur moyenne-Calculée d'après le tab. I)

Les écarts qui apparaissent s'expliquent par les différentes méthodes de mesure utilisées (3 types d'appareils) et par les caractéristiques pressiométriques issues d'essais qui ne jouxtent pas chaque pieu d'accostage. Cette dernière remarque intéresse tout autant la distribution des moments fléchissants. La figure 7 donne, pour différentes charges latérales appliquées en tête, les résultats obtenus par le logiciel PILATE-LCPC et les valeurs mesurées à différents niveau dans le cas du pieu aval. On peut constater que les moments fléchissants maximaux calculés sont inférieurs à ceux mesurés, les écarts se situant en deça de 18\%. Cependant la profondeur où les moments maximaux sont atteints n'est pas affectée.

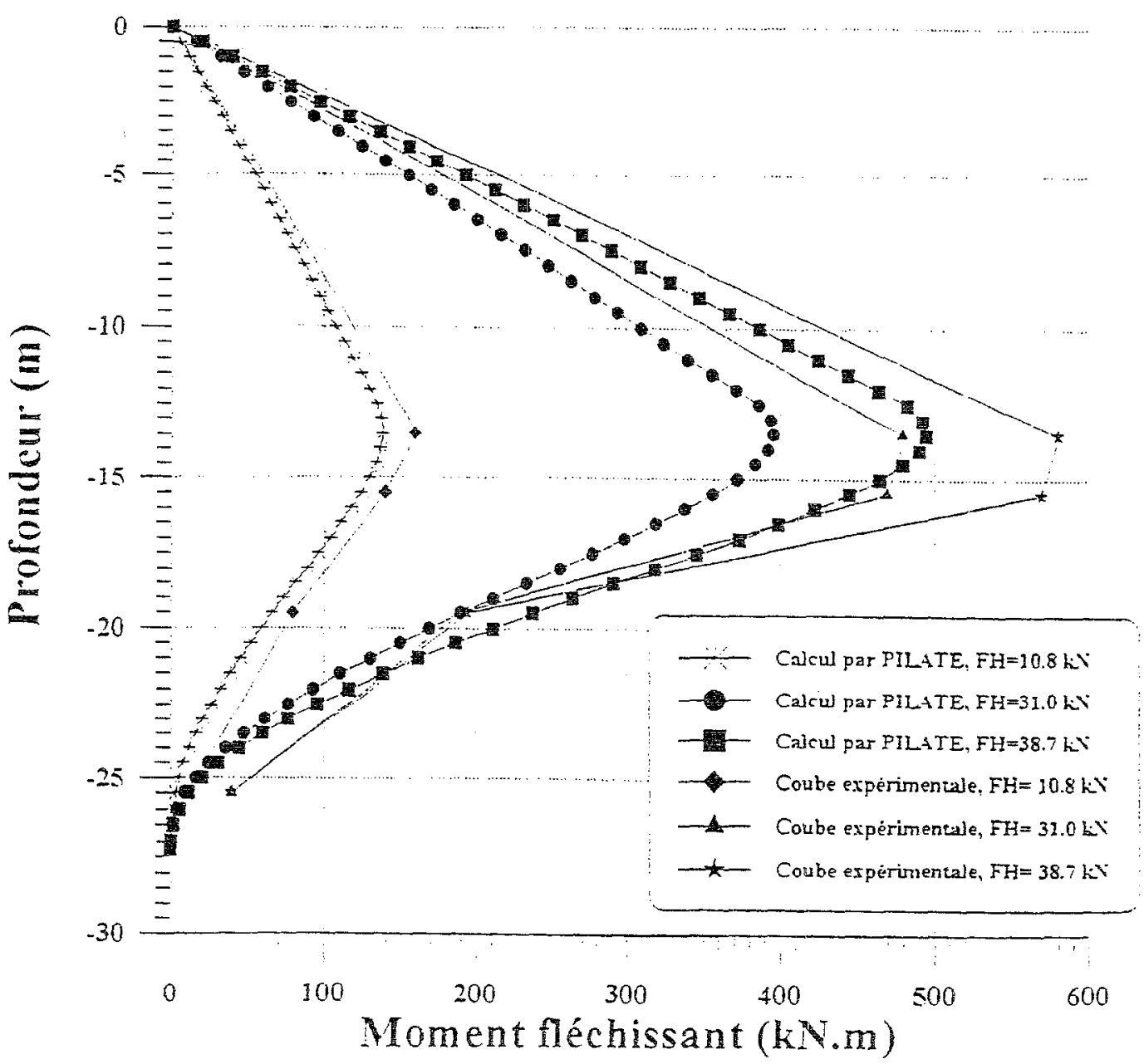

Fig. 7 : Comparaison des moments fléchissants calculés (en continu) et mesurés (valeurs ponctuelles)

Chargement appliqué : $10.8 \mathrm{kN}, 31.0 \mathrm{kN}, 38.7 \mathrm{kN}$. 


\section{Conclusions générales}

Le bilan de l'instrumentation reste relativement positif étant donné le nombre disponible de jauges opérationnelles, soit un taux de $56 \%$.

Le pieu le plus instrumenté est le pielu aval qui lors d'accostage de bateaux-sabliers est le plus sollicité. 32 jauges sur 40 sont disponibles soit un taux de $80 \%$. Ce pieu parâit le plus apte à poursuivre des essais en vraie grandeur.

Les pieux d'accostage ont été sollicités pour des efforts latéraux relativement faibles (10\% de la charge de service) qui engendrent des moments fléchissants de valeur réduite. Les courbes des moments fléchissants présentent une allure tout à fait conforme à celles obtenues en général pour un pieu relativement rigide et libre en pointe. Les moments fléchissants maximaux apparaissent à une profondeur voisine de 0,5 à $1,5 \mathrm{~m}$ de profondeur (référence 0 - fond marin). Cette référence a été relevée lors des campagnes de mai et de juillet 1994. Elle reste peut-être à confirmer.

Le mode d'installation des pieux par battage a causé des dégradations au niveau des jauges de déformation. II est aussi à l'origine des contraintes résiduelles observées dans certaines génératrices.

Le pieu aval $\left(n^{\circ} 3\right)$ parait le plus adapté pour envisager des essais de chargements latéraux beaucoup plus importants.

\section{REEERENCES BIBLIOGRAPHIOUES}

[1] LEVACHER D., VASSELIN E., "Instrumentation et essais de pieux d'accostage du terminal sablier de Cheviré", rapport CFL No 95-001, Université de Nantes, 71p.

[2] BANGRATZ R., FRAN K R. (1981), PILATE : programme de calcul d'un pieu isolé soumis à des efforts de flexion en tête et à des poussées latérales de sol, Notice d'utilisation. 